\title{
Socio-Economic Disparities in Under-Five Child Malnutrition in Nigeria
}

\author{
Oluwabukola O. Adesuyi ${ }^{1}$, Urbanus M. Kioko ${ }^{1} \&$ Martine O. Oleche ${ }^{1}$ \\ ${ }^{1}$ School of Economics, University of Nairobi, Nairobi, Kenya \\ Correspondence: Oluwabukola O Adesuyi, School of Economics, University of Nairobi, Nairobi, Kenya.
}

Received: November 11, 2020 Accepted: July 26, 2021 Online Published: August 16, 2021

doi:10.5539/gjhs.v13n9p76 URL: https://doi.org/10.5539/gjhs.v13n9p76

\begin{abstract}
Introduction: Recent attention over the health, wellbeing and nutritional intake of children below five years of age has grown tremendously. This is mainly because these years are crucial to a child's survival, growth and development; and if not handled properly could unfavorably affect the well-being status and efficiency of the child in later adult life. The study focused on malnutrition of children under the age of five in relation to their socio-economic status. It was measured by stunting, wasting and underweight.
\end{abstract}

Methodology: Data from the Living Standards Measurement Study (LSMS)/General Household Survey (GHS) 2015/2016 Nigeria was used for analysis. Malnutrition was measured using the three anthropometric measures which are expressed in terms of Z-scores namely: Stunting: height-for-age (HAZ), Wasting: weight-for-height (WHZ) and Underweight: weight-for-age (WAZ). The socioeconomic disparities in malnutrition were checked according to gender, place of residence and geo-political zones in Nigeria. While the concentration index and curves were used to check for the magnitude of inequality in malnutrition ascribable to the socio-economic status.

Results: The percentage of children stunted was the highest with 37.8 percent, followed by the percentage of children underweight to be 20.25 and children wasted was 9.63 percent. The percentage of stunting, wasting and underweight were considerably greater in male children compare to the female children. Stunting and underweight were responsive to the household socioeconomic status. A higher percentage of children below five years of age who were stunted, wasted and underweight lived in the rural areas of Nigeria compare to the children living in the urban areas. The rate of stunting was highest in the North West with a 52.91 percent, followed by North East with 43 percent, and lowest in the South South with 20.67 percent. The concentration indices analysis revealed that stunting, wasting and underweight all had negative signs signifying concentration among the poor household children. Finally, as one moves up the ladder of the socioeconomic status, a significant fall in the rate of stunting is witnessed. Therefore, increasing the income of the poorest in a society is a sound strategy to curb the high rates of stunting in the socio-economically deprived segments of the country.

Keywords: under-five children, malnutrition, stunting, underweight, wasting, concentration index and curve

\section{Introduction}

Over time, there has been an increased attention in the area of socio-economic injustices in health with individual countries and international groups renewing their efforts to restitute the wellbeing of the less fortunate and vulnerable in the society (Wagstaff, 2000; Gwatkin, 2000).

The children, especially under-fives in Nigeria are vulnerable to many conditions and diseases which eventually lead to mortality due to their socio-economic status. The reason is not far-fetched from the inequality in socioeconomic status among Nigerians and low immune system. Some common conditions in children below five years include: Chronic Diarrhea which affects close to 1.3 million episodes. Worldwide, close to 4 million people die due to preventable conditions. Over 80\% deaths occur in Sub-Saharan countries. Worldwide 300 -500 million clinical cases of malaria are reported annually, which accounts for $80 \%$ occurring in Africa alone, resulting in about $0.7-2.5$ million deaths in Sub-Saharan Africa. It is disheartening to note that Nigeria ranks among the top 45 nations that contribute to $94 \%$ of the global life losses due to measles.

In Nigeria, measles has a dominance of $1.3-5.1 \%$ of all pediatric records. However most of these diseases and sicknesses are linked to malnutrition cases (Saleh, 2013). Poverty is a major cause of malnourishment, which has its roots from the socioeconomic status of individuals and households. 
Studies have shown the existence of an inverse relationship between socio-economic status and malnutrition, sickness, and mortality (Chaturvedi, Jarrett, Shipley, \& Fuller, 1998). There is a sizable literature documenting socioeconomic inequality in malnutrition. Zere and McIntyre (2003), carried out a research to ascertain and quantify the prevalence of inequalities in below-five baby's malnourishment, especially those attributable to socio-economic conditions in South Africa. Results showed that stunting was the major prevailing type of under-nutrition in South Africa. Its rate was found to be highly prevalent in Eastern Cape as well as some sections of the North - provinces that have highest intensity of poverty. They also found a significant pro-rich unfairness in the allocation of stunting and underweight cases. Nevertheless, wasting showed no gradients attributed to socio-economic standing. They therefore concluded that there were significant differences in under-five child malnutrition (stunting and underweight) that favored the wealthy in the society; which are unnecessary, avoidable and unjust.

Poel et al. (2007) did a study in Ghana with the aim of analyzing inequalities in children's height-for-age z-scores across the entire socioeconomic distribution and decomposes this inequality into different contributing factors using a concentration index. Findings show that malnourishment is connected to poverty, mother's education, health care and family planning and regional characteristics. Socioeconomic inequality in malnutrition is mainly associated with poverty, health care use and regional disparities. Child malnutrition in Ghana is said to be a multi-sectorial problem. The factors associated with average malnutrition rates are not necessarily the same as those associated with socioeconomic inequality in malnutrition.

Bangladesh has malnutrition as one of the principal causes of child mortality. Islam et al., (2013) did a study on Bangladesh with the aim of demonstrating the applicability of the generalized Poisson regression (GPR) model as an alternative of other statistical methods and to find some predictors of malnutrition. The study identified several significant predictors of the outcome variable namely mother's education, father's education, wealth index, sanitation status, source of drinking water, and total number of children ever born to a woman. Rahman et al., (2016) examined the association between low birth weight (LBW) and malnutrition with findings revealing that the prevalence of malnutrition was remarkably higher in children with LBW than those with normal birth-weights. While, Thang and Popkin (2003) showed that children from rural homes, poor families, and cultural inferior backgrounds were considerably more probable to be under-nourished as equated to those residing in the urban parts and the babies of the rich families.

Kandala et al., (2011) gave a different twist to the study of undernourishment in the DRC by examining spatial variation in under-five malnutrition with flexible geo-additive semi-parametric mixed model while simultaneously controlling for spatial dependence and possibly nonlinear effects of covariates within a simultaneous, coherent regression framework based on Markov Chain Monte Carlo techniques. Results showed that malnutrition was significantly higher in rural areas compared to urban centers and this difference persisted after multiple adjustments. Also childhood malnutrition was more pronounced in all provinces of the DRC, after accounting for the location's effects, geographic differences were significant.

Poel et al., (2008) carried out a study involving 47 developing countries, Nigeria included. They aimed at evaluating the socioeconomic imbalances in malnourishment of babies, to provide proof for correlation between socioeconomic imbalance and the mean level of under-nutrition, and to attract attention to diverse trends of socioeconomic unfairness in malnourishment. They utilized data from the 47 DHSs that had data on the nutritional cases of minors not exceeding 5 years. Results showed that in nearly every country, stunting had disproportionate impacts on the poor. The concentration index was notable in every country, except Madagascar. Socioeconomic unfairness in stunting was highest in the Latin America as well as the Caribbean region. Wasting was overlay concentrated in the poorest regions. However, socioeconomic imbalance was less as compared with stunting. For nearly $1 / 3$ of nations, socioeconomic imbalance was negligible. A comparison of the mean stunting and wasting cases were made in relation to WHO child development standards and NCHS development standards. For the two pointers of malnourishment, the mean rate was more when the new WHO basic guidelines were adhered to. Nonetheless, socioeconomic imbalance was fairly comparable with the two distinct development standards.

A similar study by Uthman (2009) was carried out in Nigeria to determine the magnitude of inequalities for below-five years suffering from malnutrition, which is as a result of socio-economic status. The data on 4187 babies below 5 years came from the Nigeria Demographic and Health Survey (2003). The study used family possession index as a proxy of the socio-economic situation. Socio-economic variation in acute minority malnutrition evaluated through the application of the "extended illness concentration and achievement indices". The findings showed significant pro-rich unfairness in spreading stunting. The South-East (SE) and South West (SW) regions recorded few mean rates of childhood malnourishment; however, large disparities occurred among 
the poor families versus the rich. Comparatively, North-east (NE) and North West (NW) recorded narrow gaps among the poor versus the rich on childhood malnourishment. He concluded that, there were substantial contrasts in malnutrition of children below 5 years that favor the rich in the society in every geopolitical locality.

Uthman (2009) also did another study with an objective to measure and decompose the socio-economic unfairness in under-nutrition of minors in Nigeria. Personal statistical records were formulated to represent a national sample of 4187 babies below five years in Nigeria. The family's socio-economic condition was determined by applying the principal component evaluation technique. The concentration index (CI) of under-nourishment in babies determined the socio-economic injustices and dissembled into its causal variables. The enormous contributors of inequality in malnourishment of children were family's economic conditions (31\%), healthcare service indicator $(17 \%)$, motherly education $(13 \%)$ as well as appropriate hygiene at $11 \%$. The findings further showed that lactation length (8\%), geopolitical areas $(8 \%)$ and place of living $(5 \%)$ additionally proved to be reasonable cause of the assessed inequality. Therefore, in its conclusion, socio-economic disparity in childhood malnourishment in Nigeria was caused by health logistics and variables outside the jurisdiction of health authorities and care delivery system.

Therefore, findings form the existing evidence show that the prevalence of under-nutrition among under-five children was significantly high and varied widely and, malnutrition has been identified as one of the principal causes of child mortality. This study therefore employed the use of Z-Score to evaluate the anthropometric status of the under-five children, while the concentration curve and index were adopted to evaluate the prevalence of inequalities in malnutrition ascribable to socioeconomic rank of under-five children in Nigeria.

In other words, malnutrition, illness and mortality increases as there is a decrease in the socio-economic status level. This is as a result of the inequality in socio-economic status, hence unfairness in healthcare sector within or amongst countries.

One indicator used by WHO for household wellbeing and determination of child survival is the diet of children under the age of five (Thomas, Strauss, \& Henriques, 1990). Infant dietary deficiency is among the leading causes of children mortality (Pelletier, Frongillo, Schroeder, \& Habicht, 1995). Infant malnourishment is a predominant community wellbeing concern in growing nations, like those in the sub-Saharan region of Africa (De Onis, 2000; Stevens et al., 2012). There are 17.6 million babies in this African region suffering from chronic malnourishment (World Bank Data, 2016). In 2015, about 7.7\% of babies were lost to malnutrition globally, 24.5\% had under-heights whereas 15\% had low weight. Sub-Saharan region and part of South-East Asia have reported many instances of malnutrition, with the former accounting for about $39.4 \%$ of under-heights, $24.9 \%$ of low weight and $10.3 \%$ lost babies below-5 years (World Health Organization [WHO], 2016).

In Nigeria, close to 2.5 million children suffer from chronic malnourishment. Statistics showed that about $7.2 \%$ of children were wasted, 32.9\% were stunted, and 19.4\% were underweight (UNICEF statistical data, 2016). Thus, given the high level of inequalities among its states and provinces, it is not unanticipated that there will exist mortality and morbidity differentials which are related to socio-economic status.

As pointed out earlier, Nigeria is made up of six main geo-political zones, and they are diverse in economic development. According to Uthman (2009) "The North East and North West regions are largely agrarian and predominantly rural. The population level of education is low. The North Central region is one-third urbanized. The South East region is slightly more urbanized than the northern regions. The South West region, which includes Lagos, the former capital is the most urbanized of the six regions. The South South region is the least urbanized of the three southern regions". Therefore the widely varied under-five malnutrition disparities found in this study is not unexpected. The southern areas of Nigeria collects around double the yearly rainwater of the north areas; hence rainfall becomes scarcer as you move up north (Kandala, Ji, Stallard, Stranges, \& Cappuccio, 2007). This informs the type of agricultural activities practiced in the various regions, with intensive farming found in the southern part of the country, while childhood malnutrition is worse in the Northern region because of the Saharan drought. The western region which is the most urbanized has the lowest rates of malnutrition. This is probably due to the level of education of parents who are likely to be more knowledgeable in child health care, enjoy better living conditions, have access to better agricultural produce, and have access to better medical care, which could translate into a lower prevalence of under-five malnutrition.

To reduce these health inequalities, the federal, state and local governments have to formulate policies authorities have to come up with policies that will help eradicate or reduce health inequalities to the barest minimum. Analysis from this study seeks to add to the existing literature by looking at disparities in under-five children in Nigeria by determining the severity of these inequalities in malnourishment of children below-five years of age which emanate from socio-economic status. 


\subsection{Objectives and Aim of the Study}

The overall purpose of the study is to find out the extent of disparities in malnutrition among children below five years of age across the country, which is related to socio-economic status and to recommend policy solutions based on findings.

\section{Theoretical Review}

A model that explains child malnutrition (under nutrition) is reviewed in this section, the framework proposed by UNICEF (1990).

\subsection{Model: United Nations Children's Fund (UNICEF) Malnutrition framework (1990)}

Unlike Davison \& Birch (2001) who focused on overweight (even though the principles apply to other forms of malnutrition), the focus here was underweight malnutrition using the conceptual framework developed by UNICEF (1990).

Therefore, UNICEF (1990) defines malnourishment/under nutrition as what happens when nutritional intake is insufficient and health unpleasant. The structure shows that the bases of malnutrition are multi-divisional, embracing caring practices, health, and food .They are also categorized as immediate, underlying, and basic, where elements at one level impact other levels.

The model showed that causes of malnutrition are related to national and community status; underlying causes includes the families and household characteristics, and the immediate causes are the individuals. Therefore these causes of malnutrition are categorized as follows: (i) Basic causes: this includes - Household access to adequate quantity and quality of resources: land, education, employment, income, technology; Inadequate financial, human, physical and social capital, Sociocultural, economic and political context; (ii) Underlying bases include: Household foodstuff uncertainty, insufficient care and nutritional practices, Unwholesome family setting and insufficient health facilities and (iii) Immediate causes include: Insufficient nutritional consumption, and Illness.

The model Sshows interplay between the variables that could lead to malnutrition. The process begins with the basic causes which include the outside or external factors such as the economic and political ideologies and influences that have impact on households and the various affairs and activities that they engage in such as the choice of health care services for the children and family as a whole, access to food that makes an household amongst others; these are summed up and categorized as underlying causes. The underlying causes in turn influence the immediate causes which are inadequate dietary intake by the children and Diseases the children are affected by. Finally, all these causes lead to a manifestation of malnutrition in children.

The UNICEF (1990) model describes causes of childhood malnutrition and that malnutrition occurs as a result of a compilation of factors including inadequate dietary intake by the child, which is due to household or family level factors such as insufficient access to food and poor water sanitation, which themselves occur as a result of societal level factors such as a lack of potential resources and limits in technology. Ultimately, the model acknowledges that people and environmental resources, economic structures, and political variables are basic contributors to malnutrition. Just like other developing countries, Nigeria has her own share of malnutrition problems, and the malnutrition situation in Nigeria tilts towards under- nutrition, hence the UNICEF (1990) framework is applicable.

\subsection{The Model}

Following the model by Rosenzweig and Schultz (1982), and UNICEF (1990) with a slight modification to the models, we assume that the child well-being production is entrenched in a utility maximizing conduct of the mother.

Therefore, the following utility function is assumed:

$$
\mathrm{U}=\mathrm{U}(\mathrm{X}, \mathrm{Y}, \mathrm{H})
$$

Where:

$\mathrm{X}=\mathrm{a}$ good that produces utility, but is not directly related to well-being of the child, also known as well-being neutral good, e.g. a child's school uniform, the mother's clothing;

$\mathrm{Y}=\mathrm{a}$ well-being-related good or behavior that produces utility to the mother and also affects the child's survival, e.g., antenatal, child delivery and post-natal care;

$\mathrm{H}=$ well-being status of a child.

The child well-being production function is given as:

$$
\mathrm{H}=\mathrm{F}(\mathrm{Y}, \mathrm{Z}, \mu)
$$


Where:

$\mathrm{Z}=$ purchased market goods and services inputs that affect the child well-being directly, such as medical care services that affects a child's well-being directly;

$\mu=$ the component of a child's well-being that is due to environmental or genetic conditions which is not influenced by parental behavior and preferences.

The mother maximizes Equation (1) given (2) subject to the budget constraint given as in Equation (3)

$$
\mathrm{I}=\mathrm{XPx}+\mathrm{YPy}+\mathrm{ZPz}
$$

Where:

$\mathrm{I}=$ exogenous income;

$\mathrm{Px}=$ price of the well-being-neutral good, $\mathrm{X}$,

Py $=$ price of well-being-related consumer good, $Y$,

$\mathrm{Pz}=$ price of child investment good, $\mathrm{Z}$.

It is important to note that from Equations (1) and (2) that the child investment good is assumed to be purchased only for the purpose of improving child health so that it enters a mother's utility function only through $\mathrm{H}$.

As stated above, a mother's production of her child's health is described in Equation (2). The child health production function has the property that it is imbedded in the constrained utility maximization behavior of the mother (Equations 1 and 3). Expressions (1)-(3) can be manipulated to yield health input demand functions of the form

$$
\begin{aligned}
& X=\text { Dx }(P x, P y, P z, I, \mu) \\
& Y=D y(P x, P y, P z, I, \mu) \\
& Z=D z(P x, P y, P z, I, \mu)
\end{aligned}
$$

The effects of changes in prices of the three goods on child health can be derived from Equations (4.1-4.3) since from Equation (2), a change in child health can be expressed as

$$
\mathrm{dH}=\mathrm{Fy} \cdot \mathrm{dY}+\mathrm{Fz} \cdot \mathrm{dZ}+\mathrm{F} \mu \cdot d \mu
$$

where,

Fy, Fz, F $\mu$ are marginal products of health inputs $\mathrm{Y}, \mathrm{Z}$ and $\mu$, respectively.

From Equation (2), the change in child health can be related to changes in respective prices of health inputs as follows

$$
\begin{aligned}
\mathrm{dH} / \mathrm{dPx} & =\mathrm{Fy} \bullet \mathrm{dY} / \mathrm{dPx}+\mathrm{Fz} \cdot \mathrm{dZ} / \mathrm{dPx}+\mathrm{F} \mu \cdot \mathrm{d} \mu / \mathrm{dPx} \\
\mathrm{dH} / \mathrm{dPy} & =\mathrm{Fy} \cdot \mathrm{d} \mathrm{d} / \mathrm{dPy}+\mathrm{Fz} \cdot \mathrm{dZ} / \mathrm{dPy}+\mathrm{F} \mu \bullet \mathrm{d} \mu / \mathrm{dPy} \\
\mathrm{dH} / \mathrm{dPz} & =\mathrm{Fy} \cdot \mathrm{dY} / \mathrm{dPz}+\mathrm{Fz} \cdot \mathrm{dZ} / \mathrm{dPz}+\mathrm{F} \mu \cdot \mathrm{d} \mu / \mathrm{dPz}
\end{aligned}
$$

where

$\mathrm{d} \mu / \mathrm{dPi}=0$, for $\mathrm{i}=\mathrm{x}, \mathrm{y}$, $\mathrm{z}$ so that in equation (6), the terms $\mathrm{F} \mu \cdot()=$.0 , since $\mu$ is a random variable unrelated to commodity prices.

The equation above shows that goods prices are associated with the well-being status of a child. The signs and magnitudes of effects of goods prices on well-being rest on

(a) Degrees of changes in demand for health inputs following price changes and on

(b) Magnitudes of the marginal products of well-being inputs.

It is fascinating to see from Equation (4.1), that changes in prices of wellbeing-neutral goods also touch child well-being through the family budget constraint. Thus, policy -makers need to know the parameters of both the child well-being production technology and the associated well-being input demands to foretell well-being effects of variations in input prices. To obtain such information, health production and input demand parameters must be assessed concurrently.

\subsection{Empirical Framework, Methodology and Estimation Techniques for Malnutrition}

To analyze and check for these interplays of variables and different categories of causes and the effect/ influence they have on malnutrition of under five children stipulated by UNICEF (1990) model, we analyzed the occurrence 
and magnitude of malnourishment in children below five years of age in Nigeria using the Z-score on anthropometric data, while the magnitude of malnutrition was analyzed by means of the concentration index.

\subsubsection{The Concentration Index (C)}

The concentration index which was developed by Kakwani 1977, 1980, is directly related to the concentration curve and it quantifies the degree of socioeconomic related inequality in a health variable (Kakwani, Wagstaff, \& van Doorslaer, 1997; Wagstaff, van Doorslaer, \& Paci, 1989).

Therefore following from Wagstaff et al. (1990), the concentration index is defined as:

$$
C=1-2 \int_{0}^{1} L_{h}(p) d p
$$

The index is bounded between -1 and 1 . For a discrete living standards variable such as this study, the concentration index (C) formula can be written as:

$$
C=\frac{2}{N \mu} \sum_{t=1}^{n} x_{i} r_{i}-1-\frac{1}{N}
$$

Where: $\mathrm{C}=$ Concentration index

$x_{i}=$ the health sector variable

$\mu=$ it's mean

$r_{1}=\mathrm{i} / \mathrm{N}$ is the fractional rank of individual $\mathrm{i}$ in the living standards distribution, with $\mathrm{i}=1$ for the poorest and $\mathrm{i}=\mathrm{N}$ for the richest.

Equation (8) can be further simplified as

$$
\mathrm{C}=\frac{2}{N \mu} \sum_{i=1}^{n} x_{i} R_{i}-1
$$

Where: $\mathrm{n}=$ the sample size

$x_{i}(i=1, \ldots n)=$ the malnutrition indicator of the $i$ th under- five child;

$\mu=$ the mean level of malnutrition; and

$R i=$ represents the socioeconomic status rank of the $i t h$ under-five child.

It can also be written as (Jenkins 1988; Kakwani 1980; Lerman and Yitzhaki 1989):

$$
C=\frac{2}{\mu} \operatorname{COV}(h, r)
$$

\subsubsection{The Concentration Curve}

The concentration curve plots the cumulative percentage of the health variable (y-axis) against the cumulative percentage of the population, ranked by living standards, beginning with the poorest, and ending with the richest (x-axis). According to Zere and McIntyre (2003), the concentration index (C) is determined from the curve of the concentration curve.

\subsubsection{Concentration Curve and Index}

To calculate the concentration index and curve we used the formula (Fuller and Lury 1977):

$$
C=\left(p_{1} S_{2}-p_{2} S_{1}\right)+\left(p_{2} S_{3}-p_{3} S_{2}\right)+\ldots \ldots+\left(p_{T-1} S_{T}-p_{T} S_{T-1}\right)
$$

Where: $p_{t}=$ the cumulative percentage of the under-five children sample ranked by socio-economic status in group $t ; S_{t}=$ corresponding concentration curve ordinate

\subsubsection{Standard Error}

In many applications, the within-group variance is assumed to be unknown, and so for this analysis, we assume the same and the variance is set at zero. The standard error of the estimator of $\mathrm{C}$ was computed using the formula given in Kakwani, Wagstaff, and van Doorslaer (1997). We define $f_{t}$ as the proportion of the sample in the th group and the fractional rank of group $t$ by

The variance of the estimator $\mathrm{C}$ is given by

$$
\operatorname{VAR}(\hat{C})=\frac{1}{n}\left[\sum_{t=1}^{T} f_{t} a_{t}^{2}-(1+C)^{2}\right]+\frac{1}{n \mu^{2}} \sum_{t=1}^{T} f_{t} \sigma_{t}^{2}\left(2 R_{t}-1-C\right)^{2},
$$

Given: $n=$ the sample size, $\sigma_{t}^{2}=$ the variance of health variable in the $t$ th group and, $\mu=$ it's mean. Therefore for 
further statistical inference the standard error for $\mathrm{C}$ above was determined using the regression model below:

$$
2 \sigma_{R}^{2}\left\lceil\frac{x_{i}}{\mu}\right\rceil=\beta_{0}+\beta_{1} R_{i}+\mu
$$

Where: the variables still maintain the same definition as in Equation (12)

$\beta_{1}$ is equal to the $\mathrm{C}$ in Equation (7) above.

The general $\mathrm{C}$ was determined (i.e. for all children below 5 years in the sample), and $\mathrm{C}$ for certain immediate, underlying, and basic causes of malnutrition, including place of residence, zones, gender etc., and the spread of malnutrition across family wealth index quintiles is assessed for every socioeconomic sub-group.

\subsubsection{The Z-Score}

This is the anthropometric approach (WHO, 1986). Following the measurement stipulated by WHO (2006), Z-score, also known as Standard Deviation (SD) score is the measure of dispersion/relative deviance of the data from the mean/median value i.e. measure of the distance between the child's value and value of the reference population. This is also known as a normal distribution, sometimes called the bell curve.

The empirical rule tells you what percentage of your data falls within a certain number of standard deviations from the mean:

- $68 \%$ of the data falls within one standard deviation of the mean.

- $95 \%$ of the data falls within two standard deviations of the mean.

- $99.7 \%$ of the data falls within three standard deviations of the mean

After the analysis, this bell curve was used to present a graphical distribution of the results. The focus was on children that were below the $-2 \mathrm{Z}$-score in the population sample.

Malnutrition is measured using the following key measurements, which were expressed in terms of Z-scores:

i. Stunting: height-for-age (HAZ) which is lower than the international standard value by more than two standard deviations;

ii. Wasting: weight-for-height (WHZ) lower than the international reference value by more than two standard deviations; and

iii. Underweight: weight-for-age (WAZ) that is more than two standard deviations below the international reference value.

For the purpose of public health significance, health policy for a country, and policy implications for this study, it is important to know the prevalence ranges of malnutrition so as to assess the magnitude of a situation as the foundation for making health policies and public health decisions. These ranges could be low, medium, high, very high, acceptable, poor, serious, critical, and very high prevalence.

Estimation of the $\mathrm{Z}$-score was done using the formula:

$$
Z=\frac{X-\mu}{\sigma}
$$

Where: Z = Z-score (HAZ, WHZ, WAZ)

$\mathrm{X}=$ Measured Value

$\mu=$ the mean or average value in the reference population

$\sigma=$ the standard deviation of the reference population (children under-five years in Nigeria)

Therefore in applying the Z-score to the measurement of malnutrition, the World Health Organization (WHO) gave the guideline in a form of reference table for the measurement of malnutrition using the $Z$-score as a value which is lower than the international standard value by more than two standard deviations.

\subsubsection{Principal Component Analysis (PCA)}

The socio-economic status was measured using the household wealth/asset index. The wealth index is a determinant of a family's aggregate living standard and was computed using the principal component analysis, which made use of the readily available information on a family's ownership (assets) of preferred resources like televisions and bicycles; materials used to construct a house; and types of water avenue and sanitation propensity.

Therefore the asset index for individual $i$ is defined as: 


$$
A_{i}=\sum_{k}\left[f_{k} \frac{\left(a_{i k}-\bar{a}_{k}\right)}{s_{k}}\right]
$$

Where:

$a_{i k}=$ the value of asset $\mathrm{k}$ for household $\mathrm{i}$

$\bar{a}_{k}=$ the sample mean

$s_{k}=$ the sample standard deviation

The wealth index is therefore grouped quintiles (poorest, poorer, poor, rich, richer).

In summary, the three anthropometric measures of malnutrition: stunting, wasting and underweight were measured using the Z-score. The concentration index and curve were used to check for the inequality in malnutrition ascribable to the socio-economic status. On the other hand, wealth index was used for the socioeconomic status of the families, and this was done using the Principal Component Analysis (PCA).

\subsection{Measurement and Definition of Variables}

The Dependent Variable which is the nutritional status/Malnutrition was measured using the three anthropometric measures which are expressed in terms of Z-scores namely: Stunting: height-for-age (HAZ), Wasting: weight-for-height (WHZ) and Underweight: weight-for-age (WAZ). The independent variables included the Socio-economic status which is expressed as the wealth index. The socioeconomic disparities in malnutrition were checked according to gender, place of residence, and zones in Nigeria. Table 1 provides a summary of definitions and measurements of the variables used in the estimations

Table 1. Variable Definitions, Measurement and Expected Effects

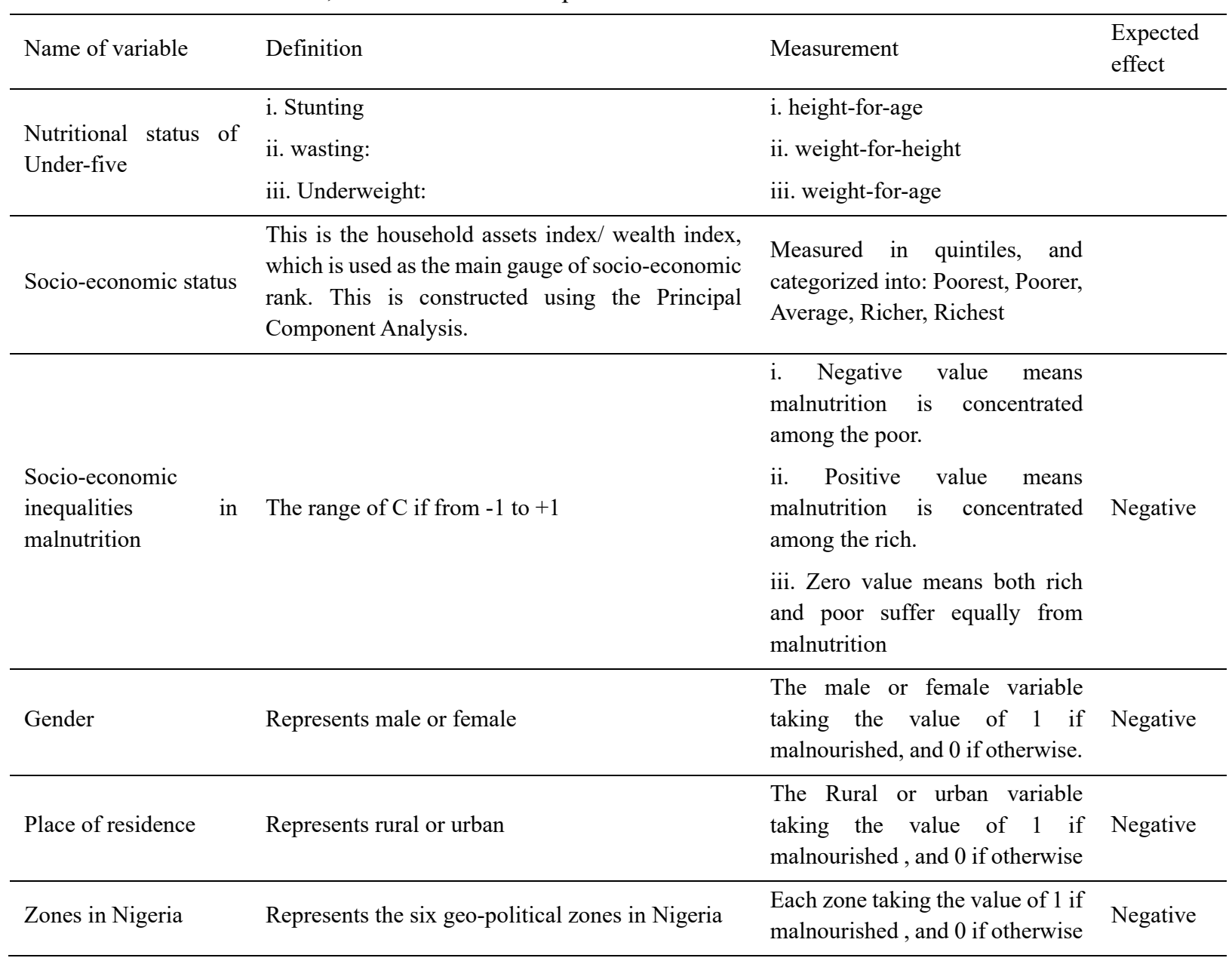

Source: Author's Analysis. 


\subsection{Data Sources}

Analysis of this study was done using data from the Living Standards Measurement Study (LSMS)/General Household Survey (GHS) 2015/2016 Nigeria data. The Nigerian General Household Survey (GHS) was implemented in collaboration with the World Bank Living Standards Measurement Study (LSMS) team as part of the Integrated Surveys on Agriculture (ISA) program and was revised in 2010 to include a panel component (GHS-Panel). The objectives of the GHS-Panel include the development of an innovative model for collecting agricultural data, inter-institutional collaboration, and comprehensive analysis of welfare indicators and socio-economic characteristics. The GHS-Panel is a nationally representative survey of 5,000 households, which are also representative of the geopolitical zones (at both the urban and rural level).

Data collection was done by groups comprising of two to four questioners, a person entering the data and a supervisor. Team number varied from state to state which was dependent on the size of the sample or the Enumeration areas chosen. It took between 20-30 days for the data collection by the teams for each of the post-planting and post-harvest visits. The data collection for the GHS-panel Wave three was done in two calls namely post=planting and post-harvest, while a tracking stage was conducted for those households that had moved from their previous locations after the preceding visit.

From the 36 states and the federal capital territory of Nigeria, sixty Enumeration Areas or primary sampling units were chosen, amounting to 2220 in total nationally, while using the multi-stage stratified sample design.

Every sampling unit had 10 families, thereby amounting to the sample size of 22,200 households. 5000 families were chosen from the 22,000 for the panel component, while 4916 families finished their interviews in the first wave. However, because some had relocated and were not reachable between the period of the first and the third wave visit, the sample size dropped slightly to 4581 families for wave 3 being a panel survey by nature. Data on 4,561 children that were below five years of age (0-59 months) were derived from the household data. These were the figures used for analysis in this study.

\section{Presentation of Results}

\subsection{Descriptive Statistics}

After the construction of the PCA, the wealth group was organized into five quintiles, representing five socioeconomic groups in Nigeria namely; Poorest, Poorer, Average, Richer, Richest. The analysis showed that the poorest quintile was the largest group in Nigeria with 24.53 percent of the population. This was closely followed by the poorer quintile with 21.27 percent, while the poor which is like the average in the country were 19.73 percent of the population. The richer and richest households in Nigeria were on the other hand 16.78 percent and 16.68 percent respectively.

\subsection{Malnutrition Z-Score Analysis}

\subsubsection{Analysis for Underweight, Stunting, and Wasting}

The Z-score analysis found the percentage of children stunted (HAZ) to be the highest with 37.8 percent, while the percentage of children not stunted are 62.23 percent. This was followed by the number of children that were underweight (WAZ) with a percentage of 20.25 and 79.75 percent of the children not underweight. Wasting (WHZ) was the lowest category with 9.63 percent of children wasted and 90.37 percent of the children not wasted.

It was observed that the distribution on the bell curve is skewed more towards the left hand side of the mean, and a number of the under-five children fell below the $-2 \mathrm{Z}$-score. This further confirmed the 37.8 percent of the stunted under-five children reported. The graph also showed the distribution of wasting in under-five children to be fairly normal, i.e. bell shaped, with majority of the children within the 95 percent bracket on the graph, and a few below the $-2 \mathrm{Z}$-score. For the underweight, the bell curve showed the distribution of children below the age of five was skewed more towards the left side of the mean, and quite a number of the children fall below the $-2 \mathrm{Z}$-score. This ascertained that the analysis of 20.25 percent of the under-five children were underweight.

Also going by the classification, we observed that the percentage of stunted children (37.77) percent fell in the category WHO interprets as high prevalence. In other words, Nigeria has a high prevalence of stunted children. Also, the percentage of children underweight (20.25 percent) fell under the classification of high prevalence, meaning also that there is a high incidence of underweight children in Nigeria. However, the 9.63 percent for wasted children fell on the borderline of poor and serious prevalence classification.

\subsubsection{Analysis by Gender}

The analysis showed that the percentage of stunting was considerably greater in male children compare to the 
female children ( 39.88 vs. 35.59 percent; $\chi^{2}=6.1, p=0.014$ ). Also the rates of wasting were higher in male children compare to the female children with 10.37 vs. 8.87 percent $\left(\chi^{2}=3.7525 ; P=0.053\right)$, and the same was the situation with underweight with males' rate higher than the females with 23.24 vs. 17.11 percent $\left(\chi^{2}=17.5210, P=\right.$ 0.000 ). Given the level of statistical significance, underweight was observed to be more statistically significant compare to wasting and stunting, which were significant at five and one percent levels.

\subsubsection{Analysis of Malnutrition by Socio-Economic Quintiles}

Further analysis of the three states of child malnutrition was done using the wealth quintiles the results revealed that stunting and underweight were responsive to the household socioeconomic status. The higher the wealth or the richer the household gets, the less stunted and underweight the children. However, wasting did not appear to be so sensitive to changes in the socioeconomic status. Majority of the children that were stunted and underweight fell within the first three poor wealth quintiles, while few fall among the last two wealth quintiles which were for the rich.

\subsubsection{Analysis of Malnutrition by Place of Residence}

Analysis of the three states of malnutrition by the areas or residence of the children showed that a higher percentage of children below five years of age who were stunted, wasted and underweight (40.41, 10.23, and 21.91 respectively) lived in the rural areas of Nigeria compare to the children living in the urban areas of the country (32.41, 8.45, 16.81 respectively).

The analysis of the three categories of malnutrition across the six geopolitical zones found in Nigeria showed that the rate of stunting was highest in the North West with a 52.91 percent, followed by North East with 43 percent, and lowest in the South South with 20.67 percent. The results also showed that North West had the highest number of children with wasting with a percentage of 11.99 , followed by North East with a rate of 9.19 percent, and the lowest number of children with wasting were found in the North Central with 7.09 percent, South South 7.7 percent and South East 7.88 percent. Wasting however, did not show substantial socioeconomic differentials among the zones. Similarly, North West zone also had the highest percentage of underweight children with a rate of 28.8 percent, also closely followed by North East with a rate of 23.3 percent. Meanwhile the zones with the lowest rates were again the South South and the North Central with the rates of 10.7 and 11.2 percent respectively.

It is interesting to know that the rate of the three types of child malnutrition had a wide spread of geographical variations, with the highest found mostly in the North West and North East zones and sadly these are also the two zones with the highest poverty rates in the country, based on the poverty indicator of the country.

The general concentration results for stunting, wasting and underweight were $-0.1217,-0.0938$ and -0.1310 respectively. The three categories of child malnutrition indicated statistically significant inequalities which were pro-rich. This means that a greater burden of malnutrition was being bored by those children in the lowest socio-economic status. However, for wasting, this socioeconomic gradient was not witnessed, as it was not so responsive to changes in socioeconomic status.

\subsubsection{Analysis of Malnutrition on Concentration Indices}

The concentration indices analysis was done on stunting, wasting and underweight, and they all have negative signs signifying that stunting, wasting and underweight were concentrated among the poor household children. The standard errors for the three measures of malnutrition were small, indicating that their sample means were more accurate reflections of the actual population mean. The result also indicated that stunting, wasting and underweight were significant at one percent level.

This result from the indices were plotted and it was seen that the income-associated and socioeconomic-associated inequalities were the sturdiest in stunting because the stunting concentration curves were far from the equality line, which is an indication of long-lasting undernourishment that is often related with socioeconomic deprivation.

Furthermore it was observed that socioeconomic-related inequalities were strong in underweight, which is also an indication of long-lasting undernourishment that is often related with socioeconomic deprivation figure. However, as anticipated, no palpable socioeconomic inequalities were observed in wasting because the concentration curve almost overlapped with the equality line. This is because income has little effect on conditions that usually precipitate wasting, such as diseases and unexpected environmental factor.

\subsubsection{Concentration Index Analysis by Geopolitical Zones}

The results from the analysis showed that stunting has equal division among the zones, with half of the zones having stunting concentrated among the poor and half having stunting concentrated among the rich (which is against apriori knowledge). The pro-rich concentration indices were significant at 5 and 1 percent level of 
significance, while South West zone was not significant, which showed that it did not exhibit income-related inequalities. The remaining three zones (South East, North East and North West) were concentrated among the rich. However, the pro-poor concentration indices were statistically not significant; in other words, they do not exhibit income-related inequalities.

Wasting was concentrated among the rich in the North Central, South East and South West zones; however, their concentration indices were also not statistically significant. While wasting was concentrated among the poor in the South South, North West, and North East with their concentration indices not statistically significant, except for the North West zone, which was significant at one percent level of significance, implying that the inequality was income-related.

Similarly, findings also revealed that underweight had an abnormally high concentration among the underprivileged in all zones; however, the pro-rich concentration indices were not statistically significant except for the South South zone which was significant at 10 percent level. This implies that the underweight concentration indices do not exhibit income-related inequalities.

\subsubsection{Concentration Index Analysis by Place of Residence}

Income-associated inequalities in stunting and underweight increased monotonically with the surge in the degree of urbanization of the family's area of dwelling. In other words, the rate of malnourished children was lower in the urban areas compare to the children living in the rural areas of the country. Wasting was also observed to have a higher concentration among rural area children compare with the urban area children, although the difference between the urban and rural was not as much as the ones observed in stunting and underweight. Between the two areas of residence -i.e. the rural and urban, the poorest bore the weightiest burden of stunting, wasting and underweight. For all population groups and places of residence, wasting did not show any gradients linked to socio-economic status. This was further clarified on the concentration curves.

\section{Discussion of Results}

This research examined the socio-economic disparities in health with distinctive reference to under-five child malnutrition. Child malnutrition is a challenge in Nigeria as it is in other countries especially in the developing countries. We observed that the percentage of stunted children derived from the study i.e. 37.77 percent fell in the category WHO interprets as high prevalence. In other words, Nigeria has a high prevalence of stunted children. This suggests that there should be targeted health policies to help reduce this high prevalence. The results is consistent with the study done by Uthman (2009), which observed that there was a high dominance of stunted children in Nigeria, and also observed that as one moves up the northern part of Nigeria, there is a remarkable increase in the proportion of stunted children. This was similar to the result observed in this study with the North West and North East zones having the highest rate of stunted children. Also, the percentage of children underweight (20.25 percent) fell in the classification of high prevalence. In other words, the occurrence of underweight children in Nigeria is high. This equally calls for targeted policies by the government and other relevant organizations to reduce this high prevalence of underweight in children. However, the 9.63 percent for wasted children fell on the borderline of poor and serious prevalence classification. This equally calls for attention by the policy makers, especially since wasting is known as the global acute malnutrition.

The result of the rate of stunting, wasting and underweight between the male and female under-five children is consistent with the result from Zere (2003), where the rate of underweight, stunting and wasting were found to be greater in the male children compare to the female children. This follows the trend that can be found in many African and developing countries, where stunting, underweight and wasting are still a challenge and especially a challenge with stunting being very slow on the decline.

For children being stunted and underweight, the socioeconomic status of a household is perceived to have a greatly substantial influence on the probability of the children. The inverse correlation that is seen between families' socioeconomic status and stunting has been well proven in the literature (Zere, 2003).

Taking a leave from other studies (Zere \& McIntyre, 2003; Mosley \& Chen, 1984), relying on global averages alone can conceal information and could be misleading when used to formulate policies. Therefore, breaking it down into various gauges by socio-economic status is very important for producing valued evidence for strategy decisions. When the different categories of under-five malnutrition is broken down by various indicators such as geographic, demographic and socio-economic status; asides from wasting, the rate of disparity in Under-five malnutrition is very much pronounced.

Disparities in the areas of residence is to some extent attributable to rural-urban differential in income disparities between places of residence, with greater rates of underweight and stunting in the rural than in the urban. The 
concentration indices for underweight, stunting and wasting show that they are concentrated among the poor in both rural and urban areas of residence, even though the pro-rich indices were highest among the rural dwellers. However, it should be noted that while stunting and underweight were higher with the poor in the rural areas, the same goes for the poor in the urban areas. Therefore, there is a need for targeting policies and other ways of combating utter poverty in the urban areas, and not just focus on the rural areas alone. This finding is consistent with other research works (Menon, Ruel, \& Morris, 2000; Bradley, Stephens, Harpham, \& Cairncross, 1992; Haddad, Ruel, \& Garrett, 1999; Basta, 1977; Zere, 2003) which found the existence of substantial concentration of ill-health amongst the metropolitan underprivileged. They found that even though "the urban population experience more variations in nutritional status, poverty, morbidity and mortality compared to rural populations, using global averages to characterize poverty and child malnutrition in urban areas may be misleading, because city averages do not capture the large heterogeneity found between social classes in urban areas". This is true for a country like Nigeria which has a high level of socioeconomic disparities.

Systematic disparities in long-established under-five undernourishment have extensive costs. Researches have shown that malnourishment contributes to a substantial decrease in lifetime earnings (Behrman \& Hoddinott, 2001). Therefore, this is likely to prolong the now great levels of income disparity in the nation.

In addition, long-standing malnourishment, particularly during the pre-school age is likely to end in irremediable harms to the child's cerebral growth. Therefore, attention on this age set is vital, as it has a significant pay-off in the future.

\section{Conclusion}

As one moves up the ladder of the socioeconomic status, a significant fall in the rate of stunting is witnessed. This implies that improvement in household's socioeconomic status will likely bring about a reduction in the probability of stunting of children. This has been highlighted by several studies that indicated that increasing the income of the poorest in a society is a sound strategy to curb the high rates of stunting in the socio-economically deprived segments of the population (Sahu et al., 2015; World Bank, 1981). Therefore, to curb disparities in stunting and underweight among regions in a country, which are likely to continue the cycle of disparities in socioeconomic status in the future, the execution of income-creating ventures and direct transfers of earnings to the underprivileged are crucial actions that must be aggressively followed by a government or policy maker. Malnourishment, particularly stunting, has a more socio-economic measurement, and hence, should be perceived in a wider context and not merely in a narrow biomedical sense.

In summary, the study found significant disparities in under-five undernourishment (underweight and stunting) which favors the rich in the society which is unnecessary, unjust and avoidable. Just like other studies have pointed out, reliance on global averages alone can be misleading (Zere and McIntyre, 2003; Mosley \& Chen, 1984), but when indicators are broken down, and analysis are conducted on specific and smaller indicators, the results are more reliable for policy formation and targets. Addressing malnutrition in under-five especially stunting, which is found to be somewhat responsive to improvement in socioeconomic status of households requires urgent attention from the policy makers or government to formulate policies that would help in improving the socioeconomic status of household, which in turn would help the reduction of malnutrition, therefore an initiative that surpasses the medical arena is needed. Finally, good nutrition is crucial to a child's survival, health and development, adversely will affect a child's survival negatively (Thomas, Strauss, \& Henriques, 1990; Pelletier et al., 1995), which may also unfavorably affect well-being status and efficiency in later adult life (Thomas, Strauss, \& Henriques, 1990). Therefore well-nourished children make better adults, who in turn make a strong and better nation.

\section{Competing Interests Statement}

The authors declare that there are no competing or potential conflicts of interest.

\section{References}

Basta, S. S. (1977). Nutrition and health in low income urban areas of the third world. Ecology of food and nutrition, 6(2), 113-124. https://doi.org/10.1080/03670244.1977.9990489

Behrman, J. R., \& Hoddinott, J. (2001). An evaluation of the impact of PROGRESA on pre-school child height (No. 583-2016-39601).

Bradley, D. J., Stephens, C., Harpham, T., \& Cairncross, S. (1992). A review of environmental health impacts in developing country cities. Washington, DC: World Bank.

Chaturvedi, N., Jarrett, J., Shipley, M. J., \& Fuller, J. H. (1998). Socioeconomic Gradient in Morbidity and Mortality in People with Diabetes: Cohort Study Findings from the Whitehall Study and the WHO 
Multinational Study of Vascular Disease in Diabetes. BMJ, 316, 100-105. https://doi.org/10.1136/bmj.316.7125.100

Davison, K. K., \& Birch, L. L. (2001). Childhood overweight: a contextual model and recommendations for future research. Obesity reviews, 2(3), 159-171. https://doi.org/10.1046/j.1467-789x.2001.00036.x

De Onis, M. (2000). Measuring nutritional status in relation to mortality / Mercedes de Onis. Bulletin of the World Health Organization: the International Journal of Public Health, 78(10), 1271-1274 https://apps.who.int/iris/handle/10665/57745

Dray-Spira, R., Gary-Webb, T. L., \& Brancati, F. L. (2010). Educational Disparities in Mortality Among Adults With Diabetes in the U.S. Diabetes Care, 33, 1200-1205. https://doi.org/10.2337/dc09-2094

Gwatkin, D.R. (2000). Health inequalities and the health of the poor: what do we know? What can we do? Bulletin World Health Organization, 78(1), 3-18. PMID: 10686729. International Health Policy Program, World Bank, Washington, DC 20433, USA.

Haddad, L., Ruel, M. T., \& Garrett, J. L. (1999). Are urban poverty and undernutrition growing? Some newly $\begin{array}{llll}\text { assembled } \quad \text { evidence. } & \text { World } & \text { 27(11), } & \text { 1891-1904. }\end{array}$ https://doi.org/10.1016/S0305-750X(99)00093-5

Islam, S. M. S., Lechner, A., Ferrari, U., Froeschl, G., Niessen, L. W., Seissler, J., \& Alam, D. S. (2013). Social and economic impact of diabetics in Bangladesh: protocol for a case-control study. BMC Public Health, 13(1), 1-9. https://doi.org/10.1186/1471-2458-13-1217

Kakwani, N., Wagstaff, A., \& van Doorslaer, E. (1997). Socioeconomic inequalities in health: Measurement, computation, and statistical inference. Journal of Econometrics, 77(1), 87-103. https://doi.org/10.1016/S0304-4076(96)01807-6

Kandala, N. B., Ji, C., Stallard, N., Stranges, S., \& Cappuccio, F. (2007). Spatial analysis of risk factors for childhood morbidity in Nigeria. American journal of tropical medicine and Hygiene, 77(4), 770-779. https://doi.org/10.4269/ajtmh.2007.77.770

Kandala, N. B., Madungu, T. P., Emina, J. B., Nzita, K. P., \& Cappuccio, F. P. (2011). Malnutrition among children under the age of five in the Democratic Republic of Congo (DRC): does geographic location matter?. BMC public health, 11, 261. https://doi.org/10.1186/1471-2458-11-261

Menon, P., Ruel, M. T., \& Morris, S. S. (2000). Socio-economic differentials in child stunting are consistently larger in urban than in rural areas. Food and Nutrition Bulletin, 21(3), 282-289. https://doi.org/10.1177/156482650002100306

Mosley, W. H., \& Chen, L. C. (1984). An analytical framework for the study of child survival in developing countries. Population and development review, 10, 25-45. https://doi.org/10.2307/2807954

Pelletier, D. L., Frongillo, E. A., Schroeder, D. G., \& Habicht, J. P. (1995). The effects of Malnutrition on Child Mortality in Developing Countries. Bulletin of the World Health Organization, 73(4), 443-448.

Poel, E. V., Hosseinpoor, A. R., Jehu-Appiah, C., Vega, J., \& Speybroeck, N. (2007). Malnutrition and the disproportional burden on the poor: the case of Ghana. International Journal for Equity in Health, 6, 21. https://doi.org/10.1186/1475-9276-6-2

Rahman, A., \& Biswas, S. (2007). Determinants of chronic malnutrition among preschool children in Bangladesh. Journal of biosocial science, 39, 161-73. https://doi.org/10.1017/S0021932006001295

Rosenzweig, M. R., \& Schultz, T. P. (1983). Estimating a household production function: Heterogeneity, the demand for health inputs, and their effects on birth weight. Journal of political economy, 91(5), 723-746.

Sahu, S. K., Kumar, S. G., Bhat, B. V., Premarajan, K. C., Sarkar, S., Roy, G., \& Joseph, N. (2015). Malnutrition among under-five children in India and strategies for control. Journal of natural science, biology, and medicine, 6(1), 18. https://doi.org/10.4103/0976-9668.149072

Saleh, K. (2013). The Health Sector in Ghana: A Comprehensive Assessment. Washington, DC: World Bank. https://doi.org/10.1596/978-0-8213-9599-8

Secrest, A. M., Costacou, T., Gutelius, B., Miller, R. G., Songer, T. J., \& Orchard, T. J. (2011). Association of Socioeconomic Status with Mortality in Type 1 Diabetes: the Pittsburgh Epidemiology of Diabetes Complications Study. Ann Epidemiol, 21, 367-373. https://doi.org/10.1016/j.annepidem.2011.02.011

Stevens, G. A., Finucane, M. M., Paciorek, C. J., Flaxman, S. R., White, R. A., Donner, A. J., ... \& Nutrition Impact 
Model Study Group. (2012). Trends in mild, moderate, and severe stunting and underweight, and progress towards MDG 1 in 141 developing countries: a systematic analysis of population representative data. The lancet, 380(9844), 824-834. https://doi.org/10.1016/S0140-6736(12)60647-3

Thang, N. M., \& Popkin, B. (2003). Child malnutrition in Vietnam and its transition in an era of economic growth. $\begin{array}{lllll}\text { Journal of Human Nutrition and } & \text { 233-244. }\end{array}$ https://doi.org/10.1046/j.1365-277X.2003.00449.x

Thomas, D., Strauss, J., \& Henriques, M. H. (1990). Child Survival, Height for Age and Household Characteristics in Brazil. Journal of Development Economics, 33, 197-234. https://doi.org/10.1016/0304-3878(90)90022-4

UNICEF statistical data. (2016).

UNICEF. (1990). Conceptual framework of the determinants of child under-nutrition.

Uthman, O. A. (2009). Decomposing socio-economic inequality in childhood malnutrition in Nigeria. Maternal \& Child Nutrition, 5(4), 358-367. https://doi.org/10.1111/j.1740-8709.2009.00183.x

Uthman, O. A. (2009). Using extended concentration and achievement indices to study socioeconomic inequality in chronic childhood malnutrition: the case of Nigeria. International journal for equity in health, 8(1), 1-8. https://doi.org/10.1186/1475-9276-8-22

Wagstaff, A. (2000). Socioeconomic inequalities in child mortality: comparisons across nine developing countries. Bulletin of the World Health Organization, 78, 19-29

Wagstaff, A., Van Doorslaer, E., \& Paci, P. (1989). Equity in the finance and delivery of health care: some tentative cross-country comparisons. Oxford review of economic policy, 5(1), 89-112. https://doi.org/10.1093/oxrep/5.1.89

World Bank Data. (2016).

World Bank. (1981). World Bank: World Development Report 1981.

World Health Organization [WHO] (1986). WHO Working Group: Use and interpretation of anthropometric indicators on nutritional status. Bulletin of the World Health Organization, 64(6), 929-941.

World Health Organization [WHO]. (2016). World Health Statistics 2016.

Wyk, V. P., \& Bradshaw, D. (2017). Mortality and socioeconomic status: the vicious cycle between poverty and ill health. PlumX Metrics, 5(9), e851-e852. https://doi.org/10.1016/S2214-109X(17)30304-2

Zere, E., \& McIntyre, D. (2003). Inequities in under-five child malnutrition in South Africa. International journal for equity in health, 2(1), 1-10. https://doi.org/10.1186/1475-9276-2-7

\section{Copyrights}

Copyright for this article is retained by the author(s), with first publication rights granted to the journal.

This is an open-access article distributed under the terms and conditions of the Creative Commons Attribution license (http://creativecommons.org/licenses/by/4.0/). 